\title{
About the Experimental Determination of the Persistence Length of Wormlike Chains of Polystyrene
}

\author{
A. Brûlet $\left(^{*}\right)$, F. Boué and J.P. Cotton \\ Laboratoire Léon Brillouin $\left({ }^{* *}\right)$, CE Saclay, 91191 Gif-sur-Yvette Cedex, France
}

(Received 15 September 1995, received in final form 1 February 1996, accepted 20 February 1996)

PACS.61.25.Hq - Macromolecular and polymer solutions; polymer melts; swelts

PACS.61.12.Ex - Neutron scattering techniques (including small-angle scattering)

\begin{abstract}
This article describes a fitting method allowing to determine the persistence length of a wormlike Kratky-Porod chain. The form factor of a flexible polymer chain is measured by means of a scattering technique. Its theoretical expression is composed of the exact calculations of des Cloiseaux for an infinite chain and of the approximated function of Sharp and Bloomfield valid for a finite chain. Two examples of the determination of $b$ illustrate this method : one describes a polyion in a semi dilute solution without salt, the other a polystyrene chain in its melt.
\end{abstract}

Résumé. - Cet article décrit une méthode permettant d'obtenir la longueur de persistance $b$ d'une chaîne de Kratky Porod. La méthode consiste à ajuster les données, mesurées par diffusion de rayonnement, avec un facteur de forme. Celui-ci provient de la fusion du calcul exact de des Cloizeaux pour une chaine infinie et de l'approximation de Sharp et Bloomfield pour une chaine finie. Elle est appliquée, avec succès, à la détermination de $b$ pour un polyion en solution semidiluée sans sel et pour une chaîne de polystyrène dans son fondu.

In the past two decades, small angle neutron scattering (SANS) has allowed [1] measurement of the form factors of polymer chains in different environnements. From such experimental form factor, we can easily determine the polymer chain conformation in various simple models, such as a cylinder or a rod shape or a Gaussian chain. But, in practice, it is often necessary to introduce a parameter in order to describe the local chain stiffness: the persistence length b. The model which is the most widely used in the analysis of the experimental data is the wormlike chain of Kratky and Porod. It accounts for a continuous transition from coil to rod. Des Cloiseaux [2] has calculated the form factor of an infinite wormlike chain as a function of the scattering vector $\mathbf{q}$. It shows that the position of the crosspoint between the behaviour of the coil $\left(q^{-2}\right)$ and that of a rod $\left(q^{-1}\right)$ at intermediate $q$ range is not clearly defined. Hence, the determination of $b$ from this position is not very accurate. Furthermore the two asymptotic $q$ ranges are far apart from each other and difficult to explore properly simultaneously. Another difficulty arises from the weakness of the scattering intensity in the asymptotic $q$ range. Finally

$\left.{ }^{*}\right)$ Author for correspondence

$\left({ }^{* *}\right)$ CEA-CNRS

(C) Les Éditions de Physique 1996 
for a finite chain, the $q^{-2}$ domain is either reduced or suppressed, increasing the difficulty of the determination of $b$.

The aim of this paper is simply to present an accurate fitting method to determine the persistence length. It corresponds to a connection of different theoretical approaches already published and is not original from this point of view. But it is, however, easy to handle. The method is illustrated by two examples of chains built on a polystyrene backbone and well known to behave as Kratky-Porod chains. The first is a polyelectrolyte chain in semi dilute solution (polystyrene sulfonate). The second corresponds to amorphous polystyrene in its melt (Sect. 2). In both cases, we have determined the persistence length of the chain using the scattering function described in Section 1. The comparison between the two form factors of both examples leads to very different results at large $q$ values. They are discussed in the last section.

\section{The Chain Form Factor}

A Kratky Porod (KP) wormlike chain [3] is a Gaussian chain which evolves continuously to a rigid rod when decreasing the distance below the persistence length $b$. Two parameters define the chain: the contour length $L$ and $b$.

The expression of the radius of gyration $R_{\mathrm{g}}$ of such a chain has been given by Benoit and Doty [4] as function of $L$ and $b$ :

$$
R_{\mathrm{g}}^{2}=b^{2}\left\{\frac{u}{3}+1+\frac{2}{u}-\frac{2}{u^{2}}[1-\exp (-u)]\right\} \quad \text { where } u=\frac{L}{b}
$$

From this equation, one could determine $b$, knowing $R_{\mathrm{g}}$ and $L$, either estimated or measured. The measurement of $R_{\mathrm{g}}$ is achieved by means of scattering, of light or neutrons, in the range of the scattering vector $q, q R_{\mathrm{g}} \leq 1$. It is perhaps easier to determine $b$ from the whole scattering curve. But for this aim, we need an analytic expression of the form factor $S_{1}(q)$ of the KP chain.

An exact calculation of $S_{1}(q)$ has been given by des Cloiseatux [2], but only in the case of an infinite chain. For $q b \leq 4$, we call this function $S_{1}^{\mathrm{C}}(q)$ : the values of $q^{2} L b S_{1}^{\mathrm{C}}(q)$ are tabulated as a function of $q b$ in reference [2]. For $q b \geq 4$, one can directly use the following asymptotic form [2] within an error (1.5\%), which is often lower than the experimental one. This asymptotic form reads:

$$
S_{1}^{\mathrm{A}}(q)=\frac{\pi}{q L}+\frac{2}{3 q^{2} b L} \quad q b \geq 4
$$

Let us note that the variation of $S_{1}(q)$ at large $q$ values allows an experimental determination of $L$, necessary to obtain $b$ from equation (1). But such a combination of equations (1) and (2) requires absolute measurements at large $q$ and the investigation of the whole $q$ range from $q R_{\mathrm{g}}<1$ to $q b>4$. This is not always possible using only one scattering technique.

In the case of finite $\mathrm{KP}$ chains, different approximations $[5,6]$ can be used. For rather long chains and small $q$ values $(q b<2)$, Sharp and Bloomfield [5] have given the following expression of the form factor:

$$
S_{1}^{\mathrm{SB}}=\left(\frac{2}{x^{2}}\right)\left(\mathrm{e}^{-x}-1+x\right)+\frac{2}{15 u}\left[4+\frac{7}{x}-\left(11+\frac{7}{x}\right) \mathrm{e}^{-x}\right]
$$

where $x=\frac{q^{2} L b}{3}$ and $u=\frac{L}{b}$. 


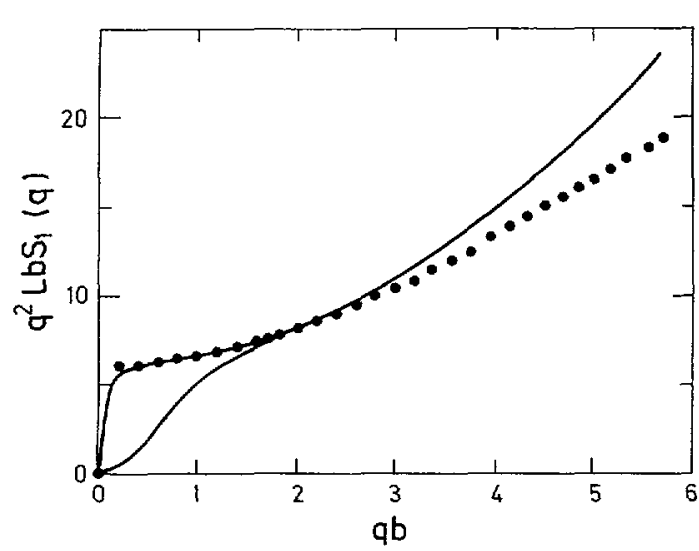

Fig. 1.

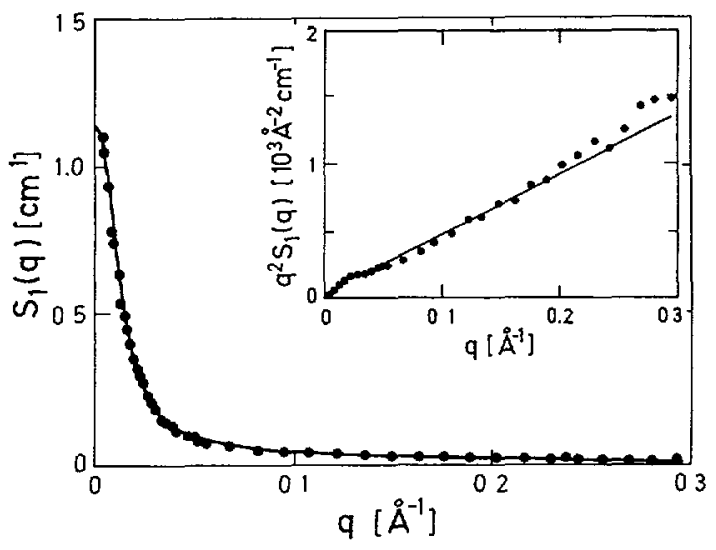

Fig. 2.

Fig. 1. - Kratky plot of the form factor $S_{1}(q)$ of a wormlike chain as a function of the dimensionless product of the scattering vector $q$ with the persistence length $b$. The full circles correspond to the calculation of des Cloizeaux [2] for an infinite chain. The full lines correspond to the approximation of Sharp and Bloomfield [5]: the upper one is obtained with a chain contour length $L=1000 b$, the lower with $L=10 b$.

Fig. 2. - Absolute intensity scattered by a polyion in a semidilute solution of sodium polystyrene sulfonate versus the scattering vector $q$. The data are those of Boué et al. [8]. The full line is a best fit to the form factor (Eq. (4)) of a wormlike chain. The fit gives a persistence length $b=65 \AA$. The insert shows the same curves in the Kratky representation which enhances the curve tail.

This equation is in a good agreement with the general expression given by Yoshizaki and Yamakawa [6]. Moreover, numerical calculations show that under the condition $L>20 b$, the values of equation (3) reach those of the des Cloizeaux function in the range $q b<2$ (see Fig. 1).

Thus we have built the form factor of the KP chain in the following way:

$$
S_{1}(q)=\left\{\begin{array}{cc}
S_{1}^{\mathrm{SB}}(q) & q b \leq 2 \\
S_{1}^{\mathrm{C}}(q) & 2 \leq q b \leq 4 \\
S_{1}^{\mathrm{A}}(q) & q b \geq 4
\end{array}\right.
$$

Representations of these functions are shown in Figure 1 using the Kratky plot, $q^{2} S(q)$ versus $q$. In this figure, the full dots correspond to the des Cloizeaux functions $S_{1}^{\mathrm{C}}$ and $S_{1}^{\mathrm{A}}$. The overlap of these asymptotic functions with the function of Sharp and Bloomfield is very good for $q b=2$. This conclusion remains valid for $L \geq 10 b$.

A closer look at equation (4) shows that the $q$ range necessary for a precise determination of $b$ is $q b \leq 1$ only. This is not surprising since in a scattering experiment the determination of a length $\ell$ is well measured in the range $q \ell \leq 1$. Nevertheless, we believe that this point has to be stressed. 


\section{Two Examples of the Determination of $b$}

The tests of the fit to equation (4) are made on the measured form factors of two kinds of polystyrene chains. In order to cover the whole $q$ range of the form factor, including $q R_{\mathrm{g}} \leq 1$, rather low molecular weights $(M \sim 100000)$ and the $q$ vector range $\left(7 \times 10^{-3}\right.$ to $\left.0.3 \AA^{-1}\right)$ were used.

For the greater values of $q$, the scattering intensity is very sensitive to the background level. Electronic and neutronic background was carefully measured and substracted from the SANS data. But the most difficult is to account for the background due to incoherent scattering. On one hand, the multiple scattering due to the large amount of incoherent scatterers (mainly hydrogen) in the sample is not calculable. On the other hand, an additional incoherent scattering arises from the random distribution of coherent scattering lengths of solvent molecules in the mixture of hydrogenated and deuterated ones [7]. We chose to prepare blanks which deliver exactly the same total incoherent scattering as the samples. Under this condition, all multiple scattering effects should be identical; the blank and the sample have the same attenuation due to incoherent scattering. Absolute measurements are made using the determination of the incident beam intensity [7]. After background corrections, the final coherent scattering cross sections are given in $\mathrm{cm}^{-1}$

2.1. Semi-Dilute Solution of Sulfonated Polystyrene PSS. - Here, we have used the results of a recent experiment [8] made in order to determine the temperature variation of the form factor of a polyion in a semi-dilute solution. The form factor of a PSS chain

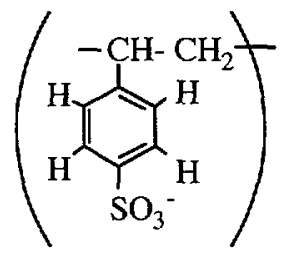

was directly determined from a SANS experiment using the zero average contrast method (ZAC) [9], which was early used for copolymer studies [9c]. Since the scattering data were not sensitive to the temperature, only the form factor measured at $30^{\circ} \mathrm{C}$ is analyzed here. The solution was a mixture of H-PSS $\left(M_{\mathrm{w}}=80000\right)$ and D-PSS $\left(M_{\mathrm{w}}=72000\right)$ in a mixture of heavy and light water containing $71 \%$ of $\mathrm{D}_{2} \mathrm{O}$ without salt. The total polymer concentration was $0.07 \mathrm{~g} / \mathrm{cm}^{3}$. The $q$ range observed was $7 \times 10^{-3}$ to $0.3 \AA^{-1}$. The background sample was a $\mathrm{H}_{2} \mathrm{O} / \mathrm{D}_{2} \mathrm{O}$ mixture with the number of protons of the solution. In the $\mathrm{ZAC}$ method the coherent intensity scattered by the polymer is very weak. Thus the blank gave the same transmission as the solution.

The best fit of the experimental data with equation (4) is shown in Figure 2. It is satisfactory and gives $L=800 \pm 50 \AA, b=65 \pm 5 \AA$ and, using equation (1), $R_{\mathrm{g}}=117 \pm 15 \AA$. These values have to be compared to those given initially in reference [8]. First, $L=1090 \pm 100 \AA$ was obtained from the slope of the straight line observed at large $q$ on the $q^{2} S(q)$ versus $q$ representation. Second, a value of $b=64 \AA$ was deduced from $L$ and from the measurement of the radius of gyration in the Guinier range, $R_{\mathrm{g}}=140 \pm 5 \AA$. Both sets of data are similar. We remark however that here the fit of the form factor does not require to perform measurements at $q b>4$ or 5 . 


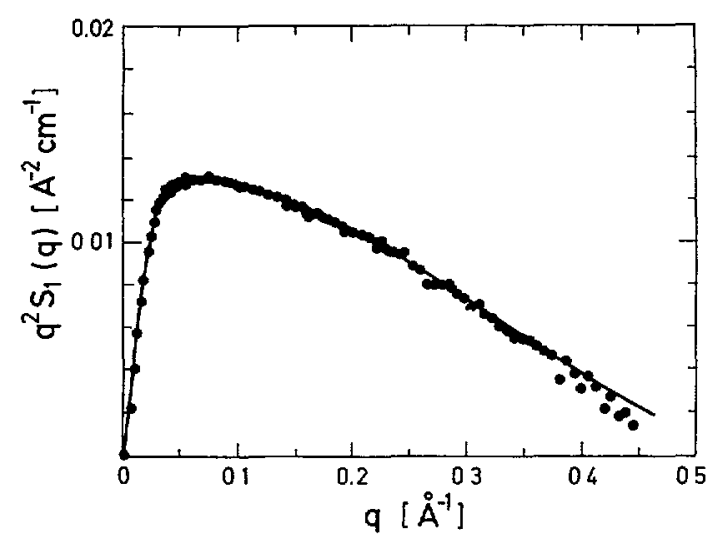

Fig. 3.

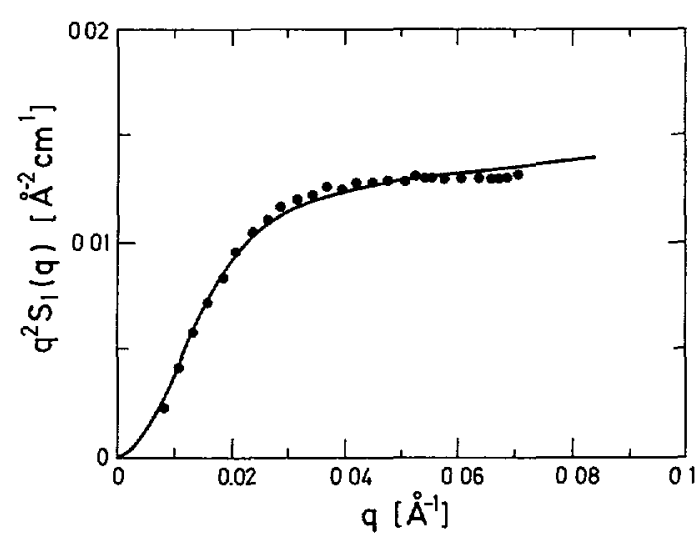

Fig. 4.

Fig. 3. - Kratky plot, $q^{2} S_{1}(q)$ versus $q$, of the absolute intensity scattered by a polystyrene chain in the melt. The full line is a best fit to a function which includes a monomer form factor (see the text). Such a decreasing function was also observed with a polystyrene in the melt (Rawiso and Boué, unpublished results).

Fig. 4. - Kratky plot of the absolute intensity scattered by a polystyrene chain in the melt. The data are those of Figure 3, truncated at $q=0.075 \AA^{-1}$. The full line is a best fit to the form factor (Eq. (4)) of the wormlike chain. The fit gives a persistence length $b=9.2 \AA$.

2.2. Melt of Polystyrene. - The method is also tested on the form factor of a classical polystyrene chain in melt. It is measured with a binary mixture of regular polystyrene (PSH)

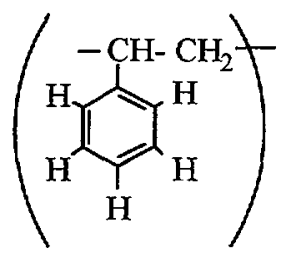

and deuterated (PSD) chains of same number of monomers. In order to measure $R_{\mathrm{g}}$ we choose rather low molecular weights $\left(M_{\mathrm{w}}=95500, M_{\mathrm{w}} / M_{\mathrm{n}}=1.17\right)$ for $\mathrm{PSD}$ and $\left(M_{\mathrm{w}}=117000\right.$, $M_{\mathrm{w}} / M_{\mathrm{n}}=1.11$ ) for PSH. The volume per volume fraction of PSD was $\Phi=0.131$. The experiments were performed on the SANS spectrometers of LLB (PAXY, PACE) in order to cover a wide $q$ range from $8 \times 10^{-3}$ to $0.45 \AA^{-1}$. The background of the $H / D$ sample was obtained with a fully protonated polystyrene sample. Its thickness was $0.632 \mathrm{~mm}$ whereas that of the sample was $0.725 \mathrm{~mm}$. Under this condition, this blank sample had the same incoherent scattering as the $\mathrm{H} / \mathrm{D}$ sample.

Figure 3 shows the chain form factor in the Kratky representation. Looking carefully at Figure 3, one can see a plateau of small extent. Its value of $0.0131 \AA^{-2} \mathrm{~cm}^{-1}$ is consistent with the value of $0.11 \Phi(1-\Phi) \AA^{-2} \mathrm{~cm}^{-1}$ often measured formely in melt [10]. At low $q, S_{1}(q)$ does not reach the $q^{-2}$ law $\left(q R_{\mathrm{g}}>4\right)$ due to the low molecular weights of the polymers.

We would like to insist more on the original behaviour observed at large $q$. There, the plateau is hidden by a decreasing function. Similar phenomena were already observed with polystyrene 
in a good solvent by Rawiso et al. [11] and were interpreted as due to the finite transverse size of the chain. In a first step, we neglect this effect and fit the experimental data only in the $q$ range below $0.075 \AA^{-1}$ (see Fig. 4). The best fit values are $L=2200 \pm 200 \AA, b=9.2 \pm 0.3 \AA$ and $R_{\mathrm{g}}=83 \pm 5 \AA$. The fit is not excellent but the $L$ and $b$ values are quite reasonable. If we take an average number of monomers of 900 , the $L$ value corresponds to a monomer length of $2.45 \AA$ close to $2.53 \AA$ the length of a monomer (a C-C-C binding). Furthermore the $b$ value can be compared to that determined from the relationship $R_{\mathrm{g}}^{2} / M_{\mathrm{w}}=2 b /\left(6 M_{\mathrm{L}}\right)$ where $M_{\mathrm{L}}$ is the linear density of the polymer, 41.2 Dalton/ $\AA$ for the polystyrene [11]. Following Fetters $e t$ al. [12], the value of $R_{\mathrm{g}}^{2} / M_{\mathrm{w}}$ for polystyrene in the melt is 0.0724 and thus an estimated value of $b$ is $8.95 \AA$. It is in a very good agreement with the value of $9.2 \AA$ obtained from our fit.

Let us now analyze the decrease of the Kratky plot observed at large $q$ (see Fig. 3 ). We introduce in the calculation of the $S_{1}(q)$ a finite transverse radius:

$$
S_{1}^{1}(q)=S_{1}(q) \Phi(q)=S_{1}(q) \exp \left(-q^{2} R_{\mathrm{a}}^{2} / 4\right)
$$

where $\Phi(q)$ is the monomer form factor and $R_{\mathrm{a}}$ the apparent radius of the chain [11]. The fit over the whole $q$ range is shown in Figure 3. We obtain $R_{\mathrm{a}}=7 \pm 0.3 \AA$. The monomer form factor does not affect the scattering curve at small $q$ and then the best fit values of $L$ and $b$ remain unchanged. This is another argument in favour of determining the persistence length with a fit in the range $q b \leq 1$.

\section{Conclusion}

From these examples, it is clearly seen that the value of the persistence length $b$ can be easily obtained from a fit of the experimental data to the chain form factor $S_{1}(q)$ of equation (4). The only requirement is the condition $L \geq 10 \mathrm{~b}$ for equation (3), which is fulfilled in most polymer studies. The great interest of this method lies in the fact that only the $q$ range $q b<1$ is required in order to obtain $b$. The asymptotic domain where $S_{1}(q) \approx(q L)^{-1}$, difficult to obtain properly, is not necessary. This conclusion is the main result of this paper.

Let us finally compare directly the data of the two examples using the normalized form factors. In Figure 5, the difference between the two curves, at large $q$ values, is striking if we consider that the scattering function in this $q$ range reflects the form factor of a monomer. As the monomers are similar, both curves should be similar too: it is clearly not the case. The form factor of the monomer for the PS in the melt appears to be important whereas it seems to be near 1 for PSS. This result for PSS is confirmed by the monomer length of $2.4 \AA$ (obtained from the $L$ value of the fit (see Fig. 5) and from an average number of monomers of 330) very similar to that of $2.45 \AA$ found for PS. The difference is more surprising considering their respective sizes: the transverse size of PS momoner is smaller than that of PSS due to the sulfonate groups. As a matter of fact, the monomer form factor can be written:

$$
\Phi(q)=k^{-2} \sum_{\imath, \jmath} k_{\imath} k_{\jmath} \cdot\left\langle\mathrm{e}^{\imath \mathbf{q}\left(\mathbf{r}_{2}-\mathbf{r}_{3}\right)}\right\rangle
$$

where $k_{2}$ is the contrast length of the $i^{\text {th }}$ atom at the position $r_{2}$ and $k$ the contrast length of the whole monomer. Thus the function $\Phi(q)=1$ found for PSS can result from a compensation of the partial form factors in equation (6). 


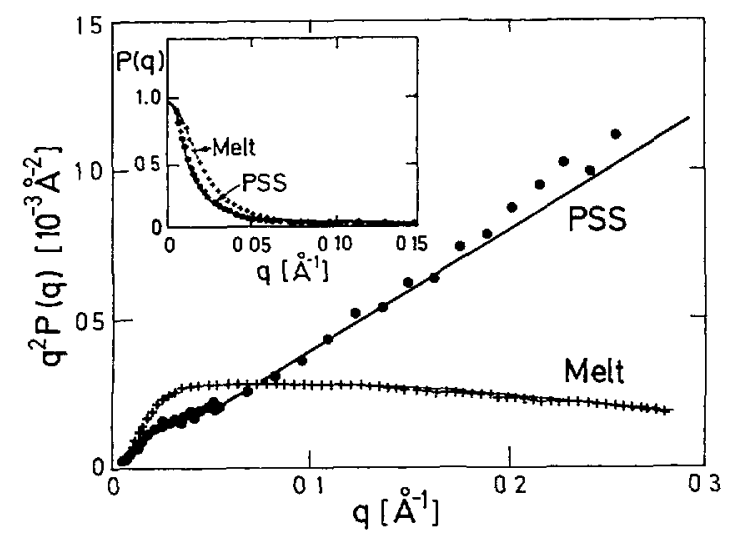

Fig. 5. - Kratky plot of the normalized scattering intensities, $P(q)=S_{1}(q) / S_{1}(0)$, for a polyion of polystyrene sulfonate in a semi-dilute solution $(\bullet)$ and for a chain of polystyrene in its melt $(+)$. The full lines are the best fits shown in Figures 2 and 3 . The interest lies in the difference which appears at large $q$ values. This difference is less impressive in the direct representation of $P(q)$ versus $q$ shown in the insert.

\section{Acknowledgments}

The authors have benefited from fruitfull discussions with M. Rawiso. They wish to thank L. Fetters for providing the up to date relationship between $R_{\mathrm{g}}$ and $M_{\mathrm{W}}$ for polystyrene and $\mathrm{J}$. Teixeira for his help in fitting the data.

\section{References}

[1] Higgins J.S. and Benoît H., "Polymers and Neutron Scattering" (Clarendon Press Oxford, 1991).

[2] des Cloizeaux J., Macromolecules 6 (1973) 403.

[3] Kratky O. and Porod G., Recl. Trav. Chim. Pays-Bas 68 (1949) 1106.

[4] Benoît H. and Doty P.M., J. Phys. Chem. 87 (1953) 958.

[5] Sharp P. and Bloomfield V.A., Biopolymers 6 (1968) 1201.

[6] Yoshizaki T. and Yamakawa H., Macromolecules 13 (1980) 1518.

[7] Cotton J.P., "Neutron, X-Ray and Light Scattering", P. Lindner, T. Zemb Eds. (Elsevier NewYork.. 1991) p. 19.

[8] Boué F., Cotton J.P., Lapp A. and Jannink G., J. Chem. Phys. 101 (1994) 2562.

[9] a) Csiba T., Jannink G., Durand D., Papoular R., Lapp A., Auvray L., Boué F., Cotton J.P. and Borsali R., J. Phys. II France 1 (1991) 381; b) Des Cloizeaux J. and Jannink G., "Polymers in solution: Their modeling and Their Structure" (Oxford University, Oxford, 1990) p. 269; c) Duval M., Picot C., Benmouna M. and Benoît H., J. Phys. France 49 (1988) 1963.

[10] Boué F., Thesis University Paris Sud, 1982.

[11] Rawiso M., Duplessix R. and Picot C., Macromolecules 20 (1987) 630.

[12] a) Fetters L., Lohse D.J., Richter D., Witten T.A. and Zirkel A., Macromolecules 27 (1994) 4639; b) Fetters L., Hadjichristidis N., Lindner J.S. and Mays J.W., J. Phys. Chem. 23 (1994) 619. 